\title{
ANÁLISIS PRELIMINAR DE PARÁMETROS GENÉTICOS PARA EL CARÁCTER DE PROLIFICIDAD EN LA OVEJA CANARIA DE PELO
}

\author{
PRELIMINARY ANALYSIS OF GENETIC PARAMETERS FOR PROLIFICACY IN THE \\ CANARY HAIRED SHEEP
}

\author{
Delgado, J.V. ${ }^{1}$, León, J.M. ${ }^{1}$, Bermejo, L.A. ${ }^{2}$, López-Gallego, F. ${ }^{3}$, Camacho, A. ${ }^{2}$ \\ y Santolaria, J. ${ }^{2}$
}

\begin{abstract}
${ }^{1}$ Departamento de Genética. Universidad de Córdoba. Córdoba. España.
${ }^{2}$ Área de Producción Animal. Departamento de Ingeniería, Producción y Economía Agraria. Universidad de La Laguna. Tenerife. Islas Canarias. España.

${ }^{3}$ Departamento de Producción Animal. Centro de Investigación Agraria. Finca la Orden. Consejería de Infraestructuras y Desarrollo Tecnológico. Junta de Extremadura. España.
\end{abstract}

\section{Palabras CLAVE ADICIONALES \\ Mejora genética. Heredabilidad. Repetibilidad.}

\section{RESUMEN}

En este trabajo se presentan los valores de los parámetros genéticos para prolificidad de la oveja Canaria de Pelo, obtenidos a partir de la utilización de un modelo animal con observaciones repetidas sobre la información generada en los núcleos de control de rendimiento cárnico de la raza. El modelo de análisis genético incluyó como efectos fijos: ganadería, año, época y número de parto; y como efectos aleatorios, el genotipo aditivo del animal y el efecto ambiental permanente.

Se utilizaron, como base de información, un total de 7657 partos registrados en 2889 animales de 5 ganaderías entre los años 1996 y 2008 , lo que supuso un promedio de 2,65 partos registrados por animal, suficiente para obtener una buena estimación del efecto ambiental permanente.

Los valores observados para la heredabilidad y para la repetibilidad fueron de 0,05 y de 0,1 respectivamente, valores dentro de los límites de la especie. También se aportan valores de las varianzas aditiva, fenotípica total y ambiental permanente.

Ésta es la primera estimación de parámetros genéticos que se realiza en esta raza con datos de campo, lo que supone un importante avance en el esquema de selección de la misma.

\section{SUMMARY}

This paper presents the values of genetic

Recibido: 30-9-09. Aceptado: 8-2-11.

\section{AdDitional KeYWORDS}

Breeding. Heritability. Repeatability.

parameters for prolificacy in the Canary Haired sheep, obtained from the use of an animal model with repeated observations on the information generated in the nucleus of meat yield control of the breed. The genetic analysis model included as fixed effects in herd, year, season and number of parturition, and as random effects, the additive genotype of the animal and permanent environmental effect. It was used as information base a total of 7657 births registered in 2889 animals from 5 herds between 1996 and 2008, which represented an average of 2.65 births registered per animal, sufficient to obtain a good estimate of permanent environmental effect. The observed values for the heritability and repeatability were 0.05 and 0.1 respectively, values within the limits of the species. Also values for additive, phenotypic and permanent environmental variances are provided. This is the first genetic parameter estimation in this breed with field data, which represents an important advance for its selection scheme.

\section{INTRODUCCIÓN}

La oveja Canaria de Pelo está adquiriendo una gran importancia en el subsector ganadero de las Islas Canarias al ocupar un área productiva muy poco explotada en los 


\section{DELGADO, LEÓN, BERMEJO, LÓPEZ-GALLEGO, CAMACHOYSANTOLARIA}

últimos años, como es la explotación ganadera ligada a los cultivos intensivos de platanera, papaya y otras producciones tropicales y subtropicales. Hoy en día la raza cuenta con casi veinte ganaderías y más de 5000 cabezas, casi todas ellas incluidas en la Asociación de Criadores de la Oveja Canaria de Pelo (OVICAN) reconocida por el Gobierno de Canarias como entidad colaboradora para la gestión del libro genealógico. Se presentan aquí los resultados del análisis genético desarrollado sobre la prolificidad de la raza, como un avance de la información genética a que están dando lugar los esfuerzos establecidos en el seno de la colaboración existente entre la Universidad de la Laguna, el Departamento de Genética de la Universidad de Córdoba (grupo AGR-218) y la asociación de criadores OVICAN.

\section{MATERIAL Y MÉTODOS}

Para la obtención de los parámetros genéticos se utilizaron las bases de datos existentes hasta este momento, con una información acumulada de 7657 partos correspondientes a 229 hembras, lo que suponía 2,65 partos controlados por oveja, en las 5 ganaderías estudiadas y conectadas genéticamente, durante el período 1996 a 2008.

Se evaluaron 3018 animales presentes en el pedigrí, de los cuales 1510 no tenían genealogía declarada y 1508 individuos disponían de información de padre y madre. De los animales evaluados genéticamente, un total de 237 eran sementales.

Para el cálculo de los valores de cría y la estimación de los parámetros genéticos, se aplicó un modelo animal con observaciones repetidas, utilizándose para ello el paquete informático MTDFREML (Boldman et al., 1995), que utiliza el algoritmo libre de derivadas REML (DFREML, Graser et al., 1987).

La ecuación del modelo de análisis fue la siguiente:

$Y_{i j k l m n p}=\mu+R_{i}+A_{j}+E_{k}+N p_{l}+U_{m}+E p_{n}+p(i j k l m n)$
Donde:

$\mathrm{Y}_{\mathrm{ijk} \text { imn }}$ : prolificidad de la oveja en el parto;

$\mu$ : media general de la población;

$R_{i}$ : efecto del rebaño (5 niveles);

$A$ : efecto del año de parto (13 niveles);

$\mathrm{E}_{\mathrm{k}}$ : efecto de la época de parto (4 niveles);

$\mathrm{Np}$ : efecto del número de parto (12 niveles);

$\mathrm{U}_{\mathrm{m}}$ : efecto genético aditivo del animal que produce el dato;

$E p_{n}$ : efecto ambiental permanente; p(ijklmn): residuo del modelo.

\section{RESULTADOSYDISCUSIÓN}

Los promedios fenotípicos de la raza se encuentran en la actualidad en torno a los 1,45 corderos nacidos por parto, constituyendo este carácter comercial un importante criterio de selección dentro del objetivo de la mejora de la productividad numérica de la raza. Los parámetros genéticos estimados para el carácter de prolificidad quedan reflejados en la tabla $\mathbf{I}$.

En primer lugar debe indicarse que al tratarse de una variable ligada a la reproducción de los animales, se aprecian unos bajos niveles de variabilidad genética aditiva $\left(\sigma^{2}{ }_{\mathrm{A}}\right)$. Las fracciones de la varianza debida a los efectos ambientales permanentes de la oveja $\left(\sigma_{\mathrm{Ep}}^{2}\right)$ también fueron pequeñas, de manera similar a lo expuesto en otras razas cárnicas (Rosati et al., 2002). Los valores de varianza ambiental permanente obtenidos en este estudio son incluso inferiores a los obtenidos por Hanford et al. (2002) en la raza Columbia que fueron de 0,03. Este hecho

Tabla I. Parámetros genéticos para prolificidad en ovino Canario de Pelo $( \pm$ error de estimación). (Genetic parameters of the prolificacy in the Canary Haired sheep breed).

\begin{tabular}{lc}
\hline Varianza genética aditiva $\left(\sigma_{\mathrm{A}}^{2}\right)$ & 0,0170 \\
Varianza ambiental $\left(\sigma_{\mathrm{E}}^{2}\right)$ & 0,2904 \\
Varianza ambiental permanente $\left(\sigma_{\mathrm{EP}}^{2}\right)$ & 0,0174 \\
Varianza fenotípica $\left(\sigma_{\mathrm{P}}^{2}\right)$ & 0,3248 \\
Heredabilidad $\left(\mathrm{h}^{2)}\right.$ & $0,050 \pm 0,016$ \\
Repetibilidad $(\mathrm{r})$ & $0,103 \pm 0,017$
\end{tabular}




\section{PARÁMETROS GENÉTICOS PARA PROLIFICIDAD EN LA OVEJA CANARIA DE PELO}

puede atribuirse a la homogeneidad climatológica y de sistemas de producción existentes en las ganaderías sobre las que se han realizado los controles de parideras.

Los valores de heredabilidad y repetibilidad en este análisis son muy similares a los obtenidos por Jurado et al. (1998) para la raza Rasa-Aragonesa y que fueron de 0,049 y 0,106 , respectivamente. Nagy et al. (1999) y Okut et al. (1999) obtuvieron valores de heredabilidad de 0,01 para cuatro razas explotadas en Hungría. Similares valores de heredabilidad fueron los obtenidos por León et al. (2005) para la raza ovina Segureña con 0,04 . Más elevados fueron los valores obtenidos por Hanford et al. (2005) para la raza Rambouillet con niveles de heredabilidad de 0,09. Finalmente, valores mucho más elevados de heredabilidad fueron los hallados por Ligda et al. (2000) para

\section{BIBLIOGRAFÍA}

Boldman, K.G., Kriese, L.A., Van Vleck, L.D., Van Tassel, C.P. and Kachman, S.D. 1995. A manual for use of MTDFREML. A set of programs to obtain estimates of variances and covariances. Department of Agriculture. Agricultural Research Service. U.S.

Graser, H.U., Smith, S.P. and Tier, B. 1987. A derivative-free approach for estimating variance components in animal models by restricted maximum likelihood. J Anim Sci, 64: 13621370.

Hanford, K.J., Van Vleck, L.D. and Snowder, G.D. 2002. Estimates of genetic parameters and genetic change for reproduction, weight, and wool characteristics of Columbia sheep. J Anim Sci, 80: 3086-3098.

Hanford, K.J., Van Vleck, L.D. and Snowder, G.D. 2005. Estimates of genetic parameters and genetic change for reproduction, weight, and wool characteristics of Rambouillet sheep. Small Ruminant Res, 57: 175-186.

Jurado, J.J., Espinosa, M.J., Valdemoros, F., Fantova, E., Ciudad, M.A., Vijil, E., Quintín, F., Alabart, J.L., Folch, J. y Sin, E. 1998. Valoración genética de reproductores para prolificidad en el esquema de selección de la UPRA carne la raza Chios y por Riggio et al. (2008) en la raza Scottish Blackface, con valores de 0,16 y 0,15 , respectivamente.

\section{CONCLUSIONES}

El bajo valor de la heredabilidad pone de manifiesto una cierta lentitud en la consecución de un progreso genético eficiente, situación que era de esperar en este tipo de caracteres. Además la baja repetibilidad nos exige utilizar la información de varios partos por animal en la evaluación de sus padres. De cualquier manera nuestros resultados muestran una correcta disposición de las bases de datos y una adecuada distribución de la información recopilada, hecho que permite la utilización de modelos de análisis avanzados como es el modelo animal con observaciones repetidas.

Aragón. Prod Ovina Caprina, 23: 201-204.

León, J.M., Barba, C., Gama, L.T., Carolino, N.P., Puntas, J., Quiroz, J. y Delgado, J.V. 2005. Parámetros genéticos de prolificidad de la oveja Segureña. Resultados preliminares. Arch Zootec, 54: 323-326.

Ligda, Ch., Gabriilidis, G., Papadopoulos, Th. and Georgoudis, A. 2000. Estimation of genetic parameters for production traits of Chios sheep using a multitrait animal model. Livest Prod Sci, 66: 217-221.

Nagy, I., Solkner, J., Komlosi, I. and Safar, L. 1999. Genetic parameters of production and fertility traits in Hungarian Merino sheep. J Anim Breed Genet, 116: 399-413.

Okut, H., Bromley, C.M., Van Vleck, L.D. and Snowder, G.D. 1999. Genotypic expression at different ages: I. Prolificacy traits of sheep. $J$ Anim Sci, 77: 2357-2365.

Riggio, V., Finocchiaro, R. and Bishop, S.C. 2008. Genetic parameters for early lamb survival and growth in Scottish Blackface sheep. J Anim Sci, 86: 1758-1764.

Rosati, A., Mousa, E., Van Vleck, L.D. and Young, L.D. 2002. Genetic parameters of reproductive traits in sheep. Small Ruminant Res, 43: 65-74. 